Published in final edited form as:

Neuroimaging Clin N Am. 2018 February ; 28(1): 43-53. doi:10.1016/j.nic.2017.09.004.

\title{
Imaging of Concussion in Young Athletes
}

Jeffrey P. Guenette, M.D. ${ }^{1,2}$, Martha E. Shenton, Ph.D. ${ }^{1,2,3,4}$, and Inga K. Koerte, M.D. ${ }^{2,4,5}$

${ }^{1}$ Department of Radiology, Brigham \& Women's Hospital, Harvard Medical School, Boston, MA

2Psychiatry Neuroimaging Laboratory, Department of Psychiatry, Brigham \& Women's Hospital, Harvard Medical School, Boston, MA

${ }^{3}$ VA Boston Healthcare System, Brockton Division, Brockton, MA

${ }^{4}$ Department of Psychiatry, Massachusetts General Hospital, Harvard Medical School, Boston, MA

${ }^{5}$ Department of Child and Adolescent Psychiatry, Psychosomatic, and Psychotherapy, Ludwig Maximilian-University, Munich, Germany

\section{Synopsis}

Conventional neuroimaging examinations are typically normal in concussed young athletes. A current focus of research is the characterization of subtle abnormalities after concussion using advanced neuroimaging techniques. These techniques have the potential to identify diagnostic and prognostic biomarkers of concussion. In the future, such biomarkers will likely provide important clinical information regarding the appropriate time interval before return-to-play, as well as a young athlete's risk for prolonged post-concussive symptoms and long-term cognitive impairment. This review provides current results from advanced imaging techniques, with an emphasis on imaging modalities that will likely become available in the near future for the clinical evaluation of concussed young athletes.

\section{Keywords}

Concussion; Mild Traumatic Brain Injury; Head Trauma; Sports; Athletics; Pediatrics; Youth; Magnetic Resonance Imaging; Diffusion Tensor Imaging; Susceptibility Weighted Imaging

\section{Introduction}

Concussion, also known as mild traumatic brain injury (mTBI), is a clinical syndrome characterized by an immediate but transient alteration in brain function, generally caused by

Corresponding Author: Inga K. Koerte, M.D., Psychiatry Neuroimaging Laboratory, 1249 Boylston St., Boston, MA 02215, Telephone: 617-525-6105, Fax: 617-525-6150, ikoerte@bwh.harvard.edu.

Publisher's Disclaimer: This is a PDF file of an unedited manuscript that has been accepted for publication. As a service to our customers we are providing this early version of the manuscript. The manuscript will undergo copyediting, typesetting, and review of the resulting proof before it is published in its final citable form. Please note that during the production process errors may be discovered which could affect the content, and all legal disclaimers that apply to the journal pertain.

Disclosures:

Jeffrey Guenette has nothing to disclose. 
a blunt force. In young athletes, the cause is typically a direct blow to the head. The American Academy of Neurology and the American Medical Society for Sports Medicine guidelines currently indicate that there is no role for routine clinical imaging in sportsrelated concussion given that the findings are typically negative. ${ }^{1,2}$ However, many new and emerging imaging techniques demonstrate their potential diagnostic and prognostic value.

\section{Epidemiology}

Half of pediatric emergency department visits for concussion are sports-related. ${ }^{3}$

Specifically, over 170,000 children age 19 or younger are seen annually for sports and recreation-related traumatic brain injury in the United States. ${ }^{4}$ Further, over 62,000 cases of concussion occur annually in high school varsity athletes, $60 \%$ of which occur in football players. ${ }^{5}$ Moreover, concussion comprises nearly $9 \%$ of reported high school athletic injuries and $6 \%$ of collegiate athletic injuries ${ }^{6}$ and there is evidence that sports-related concussion is substantially under-reported. For example, amateur male hockey players report approximately 14 times more concussions than official reports of such injury suggest. ${ }^{7}$ The most common symptom of concussion is headache, which occurs in $93.4 \%$ of concussed high school athletes, while loss of consciousness occurs $4.6 \%$ of the time. ${ }^{8}$

Reported rates of concussion in high school sports has, in fact, increased 4.2 fold from 1997-1998 to 2007-2008, ${ }^{9}$ and this increase corresponds to a similar increased rate of concussion-related emergency department visits, which has doubled in 8 to 13 year-old children and tripled in 14 to 19 year-old children in the United States over this same time period. Of note, this increase is despite an overall decline in participation in organized team sports. ${ }^{3} \mathrm{~A}$ similar rate of increase in emergency department visits for concussion has also been reported by the Center for Disease Control for the years 2001 to 2009. ${ }^{4}$ The activities most associated with youth emergency department visits for concussion are bicycling, football, playground activities, basketball, and soccer. ${ }^{4}$ In addition, over $10 \%$ of youth emergency department visits for concussion are related to horseback riding, ice skating, golfing, all-terrain vehicle riding, and tobogganing/sledding. ${ }^{4}$

\section{Sex differences in concussion}

Although over $70 \%$ of emergency department patients with sports and recreation-related concussion visits are male, ${ }^{4}$ high school girls experience twice the rate of concussion as boys participating in similar sports. ${ }^{9}$ Even more concerning is that female high school and collegiate athletes who sustain a concussion experience more frequent cognitive impairment, display greater declines in simple and complex reaction times relative to preseason baseline levels, and they also experience more post-concussion symptoms compared to males. ${ }^{10} \mathrm{~A}$ study of children hospitalized after concussion also reports more severe symptoms in girls than in boys at presentation. ${ }^{11}$ Similar findings have been identified in a study of female soccer players who performed worse on computer-based neuropsychiatric testing after concussion than did males, ${ }^{12}$ and in a neuroimaging study of female athletes who had more severe white matter abnormalities at 6 months post-concussion than did males. ${ }^{13}$ 


\section{Considerations Regarding Timing of Imaging}

Based on physical exam, clinical anatomic imaging is performed in the acute setting if skull fracture or hemorrhage is suspected. While cognitive function typically returns to baseline in 5-7 days, ${ }^{14}$ in one study of 416 children and adolescents with concussion, $29.3 \%$ had postconcussion symptoms at 3 months, and missed over 1 week of school on average. ${ }^{15}$ Similarly, in another study of 190 children, almost $25 \%$ experienced headache, $20 \%$ fatigue, and 20\% longer thinking times at 1 month, with average sleep disturbances lasting 16 days. ${ }^{16}$ Given that these symptoms are measured on a timeline of weeks to months, it is important to develop imaging biomarkers to identify the acute or subacute phase to determine whether or not this information will have value in predicting outcome (prognosis). Thus imaging examinations performed in the chronic phase at 1 month to 3 months would more likely reveal alterations in the brain that may be useful in predicting which patients who experience post-concussion symptoms will likely suffer prolonged post-concussive symptoms or even permanent cognitive deficits.

\section{Objective of This Review}

Research on neuroimaging of concussion in young athletes is currently focused on identifying highly sensitive and quantitative measures of early concussion diagnosis, and towards accurate prognosis. The goal of these efforts is to identify imaging markers that can be used as biomarkers that provide important clinical information, including an individual's risk for prolonged post-concussive symptoms, risk for long-term cognitive deficits, and an appropriate time interval before return-to-play. This review discusses the results of advanced imaging techniques that are currently being used in neuroimaging research in youth concussion, with an emphasis on those techniques that may soon be incorporated into the clinical evaluation of concussed young athletes.

\section{Normal Anatomy and Imaging Technique}

\section{Traditional Clinical Anatomic Imaging}

The American Academy of Neurology guidelines recommend conventional clinical neuroimaging in the setting of suspected concussion in those patients who have loss of consciousness, posttraumatic amnesia, persistent Glasgow Coma Scale <15, focal neurologic deficit, evidence of skull fracture on examination, or signs of clinical deterioration. ${ }^{1}$ Neuroimaging in this context is to rule out more severe traumatic brain injury. Similarly, the American Medical Society of Sports medicine guidelines recommend traditional clinical neuroimaging only when there is concern of intracranial hemorrhage. ${ }^{2}$

Computed Tomography (CT) imaging is the first-line imaging in head injury:

- $\quad$ CT has higher spatial resolution than MRI;

- $\mathrm{CT}$ is more sensitive than MRI for the evaluation of the osseous calvarium, provides exceptional sensitivity for hemorrhage, and makes possible the detection of foreign objects inside the calvarium that would preclude MRI;

- $\quad$ CT is also cheaper, more readily available, and much faster than MRI. 
CT findings in moderate to severe brain trauma include skull base fracture, epidural hemorrhage related to laceration of the middle meningeal artery from an adjacent fracture (Figure 1), and subarachnoid hemorrhage or cerebral contusion related to brain compression and friction along the rough inner calvarial surface (Figure 2). However, the use of CT includes the risk of exposure to ionizing radiation and it lacks sensitivity for detecting diffuse white matter injury and subtle structural brain alterations. MRI is far more sensitive for detecting diffuse axonal injury, the most common injury observed in concussion (Figure $3)$.

\section{Considerations Regarding Patient Selection for Imaging}

Advanced imaging techniques are nonetheless time-consuming and expensive. Screening is thus important in considering its use in the evaluation of concussion. For example, screening based on a history of prior concussion may be appropriate given that high school football players with prior concussion have a greater risk of future concussion. ${ }^{17}$ High school athletes with a history of prior concussion are also more likely to experience on-field lossof-consciousness, anterograde amnesia, and confusion, ${ }^{18}$ and they perform more poorly on memory testing, attention, and concentration following a new concussion than do athletes experiencing their first concussion. ${ }^{12,19}$ Similarly, screening of athletes with academic problems may be appropriate to evaluate for prior concussion particularly given that high school athletes with a history of two or more concussions often perform similarly on baseline measures of concentration and attention to those recently concussed. ${ }^{20}$ Screening based on length of on-field symptoms may also be appropriate given that high school athletes with on-field mental status changes for more than 5 minutes have longer-lasting post-concussion symptoms and memory decline than those with a shorter durations of symptoms..$^{21}$ Finally, screening based on age may be appropriate given that high school athletes demonstrate prolonged memory dysfunction following concussion compared with college athletes. ${ }^{22}$ Currently, known clinical markers associated with longer time to recovery, such as self-reported cognitive decline, reaction time, and migraine symptoms, ${ }^{23}$ may also play a role in patient selection for screening using imaging.

Below we describe two imaging protocols: The first is used in the clinical setting at Boston Children's Hospital; the second is an example of a protocol used in research studies to evaluate for subtle alterations following concussion in young athletes (Box 1).

\section{Imaging Findings/Pathology}

\section{Clinical Anatomic Imaging}

As noted previously, routine clinical imaging in concussion is discouraged by both the American Academy of Neurology and the American Medical Society for Sports Medicine due to low diagnostic yield. ${ }^{1,2}$ For example, in a study of 151 youth patients with sportsrelated concussion, 8 patients had abnormal neuroimaging findings. ${ }^{24}$ Of note, this subset of patients was likely self-selected for greater-than average symptoms given that many individuals with concussion never seek medical care. 


\section{Structural Quantitative High Resolution MR Imaging}

High resolution, 3D, T1-weighted MR imaging sequences provide very good anatomic detail of the brain. Using a tool such as 3D Slicer (Surgical Planning Laboratory, Brigham and Women's Hospital, Boston, Massachusetts, USA), regions of interest within the brain can be manually segmented or parcellated. Further, tools such as FreeSurfer (Athinoula A. Martinos Center for Biomedical Imaging, Charlestown, MA, USA) and FMRIB Software Library (FSL) (Analysis Group, Oxford Centre for Functional MRI of the Brain, Oxford, England) also allow automated brain segmentation and parcellation (Figure 4).

Cortical atrophy has been observed in pediatric patients with concussion 4 months postinjury. ${ }^{25}$ Similarly, children with moderate/severe traumatic brain injury may evince widespread cortical thinning 3 years post-injury. ${ }^{26}$ Cortical thickness is also associated with memory performance. ${ }^{26}$ Also of note, there appears to be an association between accelerated decline in cortical thickness with age and a history of repetitive concussive and subconcussive mild traumatic brain injury. ${ }^{27,28}$ As for deep gray matter volumes, a study of college football players with a history of prior clinician-diagnosed concussion demonstrated smaller hippocampal volumes in players with a history of concussion compared to those without a history of concussion. ${ }^{29}$ There is also an apparent association between repeated head trauma and smaller thalamus size. ${ }^{30}$

\section{Tissue Architecture Diffusion Tensor Imaging}

Diffusion tensor imaging (DTI) is an MR method that measures diffusion of water molecules (see review by Basser and Jones). ${ }^{31}$ Diffusion measures can be calculated based on brain regions-of-interest and they can be used to analyze white matter fiber tracts (Figure 5). The physiological interpretation of DTI measures is well described. ${ }^{32,33}$ The most commonly reported diffusion measure is fractional anisotropy (FA). FA describes the likelihood of directionality of the diffusion of water. Diffusion MRI is a particularly promising technique in for evaluating the subtle structural brain alterations following concussion such as diffuse axonal injury, noted previously as the most common injury after concussion. Diffuse axonal injury can be identified and quantified using diffusion MRI. In the last few years, many studies have been published using diffusion MRI in mild traumatic brain injury (please see Shenton et al. $2012^{34}$ for a comprehensive review).

In adolescents, DTI abnormalities have been identified in the first week post-concussion. ${ }^{35}$ Moreover, white matter DTI abnormalities that are present in the first week post-concussion have been shown to persist at six months post-concussion in adult athletes. ${ }^{36}$ In young adult hockey players with a history of concussion, elevated FA in the left genu and in the anterior corona radiata are observed compared to teammates with no history of concussion, suggesting regional white matter microstructural injury following concussion. ${ }^{37}$ Similarly, regional diffusion abnormalities are observed in female athletes in the corpus callosum at 6 months post-concussion, ${ }^{13}$ and also in other white matter regions in pediatric patients 4 months post-concussion, despite partial normalization in cognitive tests. ${ }^{25}$ Taken together, these findings suggest that concussion-related white matter injuries may still be evident as clinical symptoms begin to resolve, or they may continue with persistent post-concussive symptoms. 


\section{Susceptibility-Weighted Imaging of Microhemorrhage}

Susceptibility-weighted images (SWI) are MR images designed to enhance the contrast between tissues with different magnetic susceptibilities. ${ }^{38} \mathrm{SWI}$ is particularly useful in evaluating paramagnetic properties that are present in deoxyhemoglobin and hemosiderin, the blood products of hemorrhage. SWI is sensitive for detecting brain lesions at all TBI severity levels in children ${ }^{39}$ and is more sensitive than CT and traditional clinical MRI for the detection of traumatic lesions in children following TBI ${ }^{40}$ Moreover, SWI is also useful in predicting cognitive outcomes in the initial stages post-injury, where the number and volume of microhemorrhages in children with mild TBI, and with more severe TBI, have been shown to correlate with neurological outcome. ${ }^{39}$ A proposed new quantitative technique has shown possible detection of microhemorrhage in collegiate hockey players over a single season. ${ }^{41}$ SWI measures are thus likely to be most useful for acute injury to detect micro-bleeds that often resolve over time.

\section{Regional Blood Flow Imaging}

Dynamic susceptibility contrast (DSC) MR imaging, also known as dynamic contrastenhanced (DCE) MR imaging, makes it possible to measure regional cerebral blood flow. ${ }^{42}$ DSC/DCE imaging is not frequently used in studies of concussion due to the need for administration of intravenous contrast material. Non-invasive alternatives such as arterial spin labeling (ASL) and phase contrast imaging are, however, being investigated in concussion. ${ }^{43-45}$

A phase contrast imaging study of pediatric sports-related concussion demonstrated cerebral blood flow alterations immediately following concussive trauma, with a trend for blood flow patterns to return toward baseline values at 14 days (27\%) and 30 days $(64 \%) .{ }^{46}$ Similar findings have been described in the acute phase with regional blood flow reductions correlating with post-concussive symptoms in studies with arterial spin label MR imaging. ${ }^{47,48}$ Abnormal regional cerebral blood flow has also been demonstrated in chronic pediatric mild traumatic brain injury ${ }^{49}$ and in adults 2 years following concussion. ${ }^{50}$ Further studies are, however, needed to determine whether or not non-contrast enhanced regional blood flow imaging may inform prognosis following concussion in youth athletes.

\section{Functional MR Imaging}

Blood oxygenation level-dependent (BOLD) MR imaging can be used as a proxy to evaluate cerebral blood flow changes in active brain regions. ${ }^{51}$ Accordingly, fMRI can evaluate brain region activation during the performance of specific tasks. It can also be used to evaluate regions of brain activation in the state of relaxed consciousness (i.e., also known as resting state) in order to evaluate regions associated with cognitive abilities and attention. ${ }^{52,53}$

Abnormalities in the default mode network, which is assumed to be a network of interacting brain regions with highly correlated activity, can be seen acutely in college varsity athletes in the first week following concussion. ${ }^{54}$ Focal abnormalities in the prefrontal cortex can be seen in male athletes with post-concussive symptoms. ${ }^{55}$ Moreover, there is evidence that fMRI may be predictive of recovery in concussed high school athletes. ${ }^{56}$ Long-term abnormalities in fMRI studies have also been identified in the chronic stages following 
concussion. ${ }^{57,58}$ Taken together, functional MRI may be sensitive in assessing alterations in brain function and connectivity following concussion. However, to be useful in the clinical setting, analysis techniques would need to reveal patient-specific alterations in addition to comparisons on a group level.

\section{Metabolic Imaging}

Magnetic resonance spectroscopy imaging (MRS) quantifies human tissue metabolites in vivo. A recent comprehensive review of MRS in brain imaging describes the clinical utility of MRS. ${ }^{59}$ Although MRS studies of adults with mTBI have suggested decreased regional axonal viability, ${ }^{60}$ the only study on sports-related concussion in youth did not observe any $\mathrm{N}$-acetylaspartate (NAA, neuron marker) or choline (cell membrane marker) abnormalities. ${ }^{46}$ Further studies are needed to determine the efficacy of these measures in concussion.

Positron emission tomography (PET), and single-photon emission computed tomography (SPECT), are performed by injecting a radioactive isotope bound to a metabolic tracer agent. The resolution of PET and SPECT is inferior to CT and MRI, which is problematic when trying to identify subtle changes as are expected in concussion. Moreover, radiotracer production and handling is expensive and involves intravenous injection of ionizing radiation, making PET and SPECT studies in young athletes generally not feasible at this time.

\section{Current Concerns Regarding Imaging of Sports-Related Concussion}

In addition to avoiding unnecessary radiation from $\mathrm{CT},{ }^{1,2}$ cost also needs to be considered. The high costs per identified imaging abnormality when using conventional neuroimaging to evaluate concussion ${ }^{61}$ reflect the earlier mentioned lack of sensitivity of conventional imaging techniques for the detection of subtle brain alterations. Until advanced imaging techniques become available that show efficacy in evaluating concussion and the trajectory of recovery from concussion, the American Association of Neurology and American Medical Society for Sports Medicine guidelines should be followed. In addition, screening tools to identify those patients at risk for prolonged post-concussive symptoms should be developed.

Further, post-concussive syndrome is a heterogeneous disorder and is therefore difficult to study scientifically and also difficult to assess clinically since many symptoms overlap with other disorders such as depression and post-traumatic stress disorder (PTSD). ${ }^{62}$

Additionally, research studies often rely on group statistics, but mean and median group values in imaging studies may not capture pathologic processes that may occur in different locations. More objective radiological evidence is needed which may have prognostic value in determining who is at risk for developing prolonged post-concussive symptoms. A method comparing concussed patients with a normative atlas has been proposed, ${ }^{63}$ as has a statistical method called wild boot-strapping, ${ }^{64}$ both of which attempt to identify subjectspecific abnormalities. However, much more research is needed in this area to move these methods to use in the clinic. 


\section{Diagnostic Criteria (List)}

The diagnosis of concussion remains clinical at this time. Nonetheless, advanced imaging features offer radiological evidence that may soon support early diagnosis and provide prognostic information as well as monitor the efficacy of future treatments.

\section{Differential Diagnosis (List/Callout Box)}

There is no current radiological evidence that provides a differential diagnosis of concussion, because concussion is a clinical diagnosis based on an associated head trauma. In most cases, routine clinical CT or MR imaging do not reveal any abnormalities. Future studies are, however, needed to identify concussion-specific imaging markers so as to provide radiological and not just clinical evidence of concussion. Advanced MR techniques will likely become available in the clinic in the near future, which will be useful for concussion diagnosis prognosis, and likely also the monitoring of treatment efficacy as treatments become available for those who are most likely to suffer persistent postconcussive symptoms.

\section{What the Referring Physician Needs to Know (List)}

- Routine clinical imaging is typically not indicated in sports-related concussion.

- $\quad$ Per American Association of Neurology and American Medical Society of Sports Medicine Guidelines, imaging should only be performed in concussion when there are concerns for skull fracture, intracranial hemorrhage, or other intracranial pathology based on physical exam.

- Potential diagnostic and prognostic neuroimaging biomarkers of concussion and adverse outcomes are being studied and may become clinically available over the next decade.

- $\quad$ Screening for clinical neuroimaging biomarkers will likely be essential to ensure adequate positive and negative predictive values and to maintain costeffectiveness.

- While some neuroimaging biomarkers may be appropriate in the acute phase of injury, many will likely be more appropriate in the subacute or chronic phases and timing will depend on the clinical concern.

\section{Summary}

Potential diagnostic and prognostic neuroimaging biomarkers of concussion in young athletes are currently being investigated and may become available in the near future for integration into routine clinical imaging. Replication of preliminary results and the development of techniques to quantify objectively imaging abnormalities on an individual basis, as opposed to group comparisons, are the next steps toward clinical implementation. We believe that the most promising approaches include a personalized, subject-specific profile of injuries that can be derived from multimodal neuroimaging. Accordingly, given the increasing number of reported sports-related concussions annually, neuroimaging 
biomarkers that provide critical information at an appropriate time interval before return-toschool and return-to-play, and based on an individual's risk for prolonged post-concussive symptoms, as well as on long-term cognitive deficits, would have a substantial impact on the routine clinical management of sports-related concussion.

\section{Acknowledgments}

Martha Shenton is receiving funding from NIMH and NINDS, and a VA Merit Award. Inga Koerte is supported by the NINDS.

\section{References}

1. Giza Christopher C, Kutcher Jeffrey S, Ashwal Stephen, et al. Summary of evidence-based guideline update: evaluation and management of concussion in sports: report of the Guideline Development Subcommittee of the American Academy of Neurology. Neurology. 2013; 80(24):2250-7. DOI: 10.1212/WNL.0b013e31828d57dd [PubMed: 23508730]

2. Harmon Kimberly G, Drezner Jonathan, Gammons Matthew, et al. American Medical Society for Sports Medicine position statement: concussion in sport. Clin J Sport Med Off J Can Acad Sport Med. 2013; 23(1):1-18. DOI: 10.1097/JSM.0b013e31827f5f93

3. Bakhos Lisa L, Lockhart Gregory R, Myers Richard, et al. Emergency Department Visits for Concussion in Young Child Athletes. Pediatrics. 2010; 126(3):E550-6. DOI: 10.1542/peds. 2009-3101 [PubMed: 20805145]

4. Centers for Disease Control and Prevention. Nonfatal Traumatic Brain Injuries Related to Sports and Recreation Activities Among Persons Aged $\leq 19$ Years — United States, 2001-2009. MMWR. 2011; 60(39):1337-42. [PubMed: 21976115]

5. Powell JW, Barber-Foss KD. Traumatic brain injury in high school athletes. Jama-J Am Med Assoc. 1999; 282(10):958-63. DOI: 10.1001/jama.282.10.958

6. Gessel Luke M, Fields Sarah K, Collins Christy L, et al. Concussions among united states high school and collegiate athletes. J Athl Train. 2007; 42(4):495-503. [PubMed: 18174937]

7. Williamson IJS, Goodman D. Converging evidence for the under-reporting of concussions in youth ice hockey. Br J Sports Med. 2006; 40(2):128-32. DOI: 10.1136/bjsm.2005.021832 [PubMed: 16431999]

8. Meehan William P, d'Hemecourt Pierre, Dawn Comstock R. High School Concussions in the 20082009 Academic Year Mechanism, Symptoms, and Management. Am J Sports Med. 2010; 38(12): 2405-9. DOI: 10.1177/0363546510376737 [PubMed: 20716683]

9. Lincoln Andrew E, Caswell Shane V, Almquist Jon L, et al. Trends in Concussion Incidence in High School Sports A Prospective 11-Year Study. Am J Sports Med. 2011; 39(5):958-63. DOI: 10.1177/0363546510392326 [PubMed: 21278427]

10. Broshek DK, Kaushik T, Freeman JR, et al. Sex differences in outcome following sports-related concussion. J Neurosurg. 2005; 102(5):856-63. DOI: 10.3171/jns.2005.102.5.0856 [PubMed: 15926710]

11. Blinman Thane A, Houseknecht Eileen, Snyder Caitlin, et al. Postconcussive symptoms in hospitalized pediatric patients after mild traumatic brain injury. J Pediatr Surg. 2009; 44(6):12238. DOI: 10.1016/j.jpedsurg.2009.02.027 [PubMed: 19524745]

12. Colvin Alexis, Chiang, Mullen, Jimmy, Lovell Mark, R., et al. The Role of Concussion History and Gender in Recovery From Soccer-Related Concussion. Am J Sports Med. 2009; 37(9):1699-704. DOI: 10.1177/0363546509332497 [PubMed: 19460813]

13. Chamard, Emilie, Lefebvre, Genevieve, Lassonde, Maryse, et al. Long-Term Abnormalities in the Corpus Callosum of Female Concussed Athletes. J Neurotrauma. 2016; 33(13):1220-+. DOI: 10.1089/neu.2015.3948 [PubMed: 26414496]

14. McCrea M, Guskiewicz KM, Marshall SW, et al. Acute effects and recovery time following concussion in collegiate football players - The NCAA Concussion Study. Jama-J Am Med Assoc. 2003; 290(19):2556-63. DOI: 10.1001/jama.290.19.2556 
15. Babcock, Lynn, Byczkowski, Terri, Wade Shari, L., et al. Predicting postconcussion syndrome after mild traumatic brain injury in children and adolescents who present to the emergency department. JAMA Pediatr. 2013; 167(2):156-61. DOI: 10.1001/jamapediatrics.2013.434 [PubMed: 23247384]

16. Eisenberg Matthew A, Meehan William P, Mannix Rebekah. Duration and Course of PostConcussive Symptoms. Pediatrics. 2014; 133(6):999-1006. DOI: 10.1542/peds.2014-0158 [PubMed: 24819569]

17. Schulz MR, Marshall SW, Mueller FO, et al. Incidence and risk factors for concussion in high school athletes, North Carolina, 1996-1999. Am J Epidemiol. 2004; 160(10):937-44. DOI: 10.1093/aje/kwh304 [PubMed: 15522850]

18. Collins MW, Lovell MR, Iverson GL, et al. Cumulative effects of concussion in high school athletes. Neurosurgery. 2002; 51(5):1175-9. DOI: 10.1227/01.NEU.0000031572.99927.93 [PubMed: 12383362]

19. Iverson GL, Gaetz M, Lovell MR, et al. Cumulative effects of concussion in amateur athletes. Brain Inj. 2004; 18(5):433-43. DOI: 10.1080/02699050310001617352 [PubMed: 15195792]

20. Moser RS, Schatz P, Jordan BD. Prolonged effects of concussion in high school athletes. Neurosurgery. 2005; 57(2):300-6. DOI: 10.1227/01.NEU.0000166663.98616.E4 [PubMed: 16094159]

21. Lovell MR, Collins MW, Iverson GL, et al. Recovery from mild concussion in high school athletes. J Neurosurg. 2003; 98(2):296-301. DOI: 10.3171/jns.2003.98.2.0296 [PubMed: 12593614]

22. Field M, Collins MW, Lovell MR, et al. Does age play a role in recovery from sports-related concussion? A comparison of high school and collegiate athletes. J Pediatr. 2003; 142(5):546-53. DOI: 10.1067/mpd.2003.190 [PubMed: 12756388]

23. Lau, Brian, Lovell Mark, R., Collins Michael, W., et al. Neurocognitive and Symptom Predictors of Recovery in High School Athletes. Clin J Sport Med. 2009; 19(3):216-21. [PubMed: 19423974]

24. Ellis Michael J, Leiter Jeff, Hall Thomas, et al. Neuroimaging findings in pediatric sports-related concussion. J Neurosurg-Pediatr. 2015; 16(3):241-7. DOI: 10.3171/2015.1.PEDS14510 [PubMed: 26031620]

25. Mayer Andrew R, Hanlon Faith M, Ling Josef M. Gray Matter Abnormalities in Pediatric Mild Traumatic Brain Injury. J Neurotrauma. 2015; 32(10):723-30. DOI: 10.1089/neu.2014.3534 [PubMed: 25313896]

26. Merkley Tricia L, Bigler Erin D, Wilde Elisabeth A, et al. Diffuse changes in cortical thickness in pediatric moderate-to-severe traumatic brain injury. J Neurotrauma. 2008; 25(11):1343-5. DOI: 10.1089/neu.2008.0615 [PubMed: 19061377]

27. Tremblay, Sebastien, De Beaumont, Louis, Henry Luke, C., et al. Sports concussions and aging: a neuroimaging investigation. Cereb Cortex N Y N 1991. 2013; 23(5):1159-66. DOI: 10.1093/ cercor/bhs 102

28. Koerte Inga K, Mayinger Michael, Muehlmann Marc, et al. Cortical thinning in former professional soccer players. Brain Imaging Behav. 2016; 10(3):792-8. DOI: 10.1007/ s11682-015-9442-0 [PubMed: 26286826]

29. Singh, Rashmi, Meier Timothy, B., Kuplicki, Rayus, et al. Relationship of collegiate football experience and concussion with hippocampal volume and cognitive outcomes. JAMA. 2014; 311(18):1883-8. DOI: 10.1001/jama.2014.3313 [PubMed: 24825643]

30. Bernick, Charles, Banks Sarah, J., Shin, Wanyong, et al. Repeated head trauma is associated with smaller thalamic volumes and slower processing speed: the Professional Fighters' Brain Health Study. Br J Sports Med. 2015; 49(15):1007-11. DOI: 10.1136/bjsports-2014-093877 [PubMed: 25633832]

31. Basser Peter J, Jones Derek K. Diffusion-tensor MRI: theory, experimental design and data analysis - a technical review. NMR Biomed. 2002; 15(7-8):456-67. DOI: 10.1002/nbm.783 [PubMed: 12489095]

32. Basser PJ, Pierpaoli C. Microstructural and physiological features of tissues elucidated by quantitative-diffusion-tensor MRI. J Magn Reson B. 1996; 111(3):209-19. [PubMed: 8661285]

33. Wheeler-Kingshott Claudia AM, Cercignani Mara. About "axial" and "radial" diffusivities. Magn Reson Med. 2009; 61(5):1255-60. DOI: 10.1002/mrm.21965 [PubMed: 19253405] 
34. Shenton ME, Hamoda HM, Schneiderman JS, et al. A review of magnetic resonance imaging and diffusion tensor imaging findings in mild traumatic brain injury. Brain Imaging Behav. 2012; 6(2): 137-92. DOI: 10.1007/s11682-012-9156-5 [PubMed: 22438191]

35. Chu Z, Wilde EA, Hunter JV, et al. Voxel-Based Analysis of Diffusion Tensor Imaging in Mild Traumatic Brain Injury in Adolescents. Am J Neuroradiol. 2010; 31(2):340-6. DOI: 10.3174/ ajnr.A1806 [PubMed: 19959772]

36. Henry Luke C, Tremblay Julie, Tremblay Sebastien, et al. Acute and Chronic Changes in Diffusivity Measures after Sports Concussion. J Neurotrauma. 2011; 28(10):2049-59. DOI: 10.1089/neu.2011.1836 [PubMed: 21864134]

37. Orr Catherine A, Albaugh Matthew D, Watts Richard, et al. Neuroimaging Biomarkers of a History of Concussion Observed in Asymptomatic Young Athletes. J Neurotrauma. 2016; 33(9):803-10. DOI: 10.1089/neu.2014.3721 [PubMed: 26413910]

38. Haacke E, Mark Xu, Yingbiao Cheng, Yu-Chung N, et al. Susceptibility weighted imaging (SWI). Magn Reson Med. 2004; 52(3):612-8. DOI: 10.1002/mrm.20198 [PubMed: 15334582]

39. Beauchamp Miriam H, Beare Richard, Ditchfield Michael, et al. Susceptibility weighted imaging and its relationship to outcome after pediatric traumatic brain injury. Cortex. 2013; 49(2):591-8. DOI: 10.1016/j.cortex.2012.08.015 [PubMed: 23062584]

40. Beauchamp Miriam H, Ditchfield Michael, Babl Franz E, et al. Detecting Traumatic Brain Lesions in Children: CT versus MRI versus Susceptibility Weighted Imaging (SWI). J Neurotrauma. 2011; 28(6):915-27. DOI: 10.1089/neu.2010.1712 [PubMed: 21501069]

41. Helmer Karl G, Pasternak Ofer, Fredman Eli, et al. Hockey Concussion Education Project, Part 1. Susceptibility-weighted imaging study in male and female ice hockey players over a single season. J Neurosurg. 2014; 120(4):864-72. DOI: 10.3171/2013.12.JNS132093 [PubMed: 24490839]

42. Conturo Thomas E, Akbudak Erbil, Kotys Melanie S, et al. Arterial input functions for dynamic susceptibility contrast MRI: requirements and signal options. J Magn Reson Imaging JMRI. 2005; 22(6):697-703. DOI: 10.1002/jmri.20457 [PubMed: 16261571]

43. Williams DS, Detre JA, Leigh JS, et al. Magnetic resonance imaging of perfusion using spin inversion of arterial water. Proc Natl Acad Sci U S A. 1992; 89(1):212-6. [PubMed: 1729691]

44. Spilt, Aart, Box Frieke, MA., van der Geest Rob, J., et al. Reproducibility of total cerebral blood flow measurements using phase contrast magnetic resonance imaging. J Magn Reson Imaging JMRI. 2002; 16(1):1-5. DOI: 10.1002/jmri.10133 [PubMed: 12112496]

45. Haller, Sven, Zaharchuk, Greg, Thomas David, L., et al. Arterial Spin Labeling Perfusion of the Brain: Emerging Clinical Applications. Radiology. 2016; 281(2):337-56. DOI: 10.1148/radiol. 2016150789 [PubMed: 27755938]

46. Maugans Todd A, Farley Chad, Altaye Mekibib, et al. Pediatric Sports-Related Concussion Produces Cerebral Blood Flow Alterations. Pediatrics. 2012; 129(1):28-37. DOI: 10.1542/peds. 2011-2083 [PubMed: 22129537]

47. Shu-Ping, Peng, Yi-Ning, Li, Jun, Liu, et al. Pulsed arterial spin labeling effectively and dynamically observes changes in cerebral blood flow after mild traumatic brain injury. Neural Regen Res. 2016; 11(2):257-61. DOI: 10.4103/1673-5374.177733 [PubMed: 27073378]

48. Che-Ming, Lin, Ying-Chi, Tseng, Hui-Ling, Hsu, et al. Arterial Spin Labeling Perfusion Study in the Patients with Subacute Mild Traumatic Brain Injury. PloS One. 2016; 11(2):e0149109.doi: 10.1371/journal.pone.0149109 [PubMed: 26871696]

49. Wang, Yang, West John, D., Bailey Jessica, N., et al. Decreased Cerebral Blood Flow in Chronic Pediatric Mild TBI: An MRI Perfusion Study. Dev Neuropsychol. 2015; 40(1):40-4. DOI: 10.1080/87565641.2014.979927 [PubMed: 25649779]

50. Yulin, Ge, Patel Mayur, B., Chen, Qun, et al. Assessment of thalamic perfusion in patients with mild traumatic brain injury by true FISP arterial spin labelling MR imaging at 3T. Brain Inj. 2009; 23(7):666-74. DOI: 10.1080/02699050903014899 [PubMed: 19557570]

51. Vitte E, Derosier C, Caritu Y, et al. Activation of the hippocampal formation by vestibular stimulation: a functional magnetic resonance imaging study. Exp Brain Res. 1996; 112(3):523-6. [PubMed: 9007554] 
52. Hampson, Michelle, Driesen Naomi, R., Skudlarski, Pawel, et al. Brain connectivity related to working memory performance. J Neurosci Off J Soc Neurosci. 2006; 26(51):13338-43. DOI: 10.1523/JNEUROSCI.3408-06.2006

53. Kelly AM, Clare Uddin, Lucina Q, Biswal Bharat B, et al. Competition between functional brain networks mediates behavioral variability. NeuroImage. 2008; 39(1):527-37. DOI: 10.1016/ j.neuroimage.2007.08.008 [PubMed: 17919929]

54. Militana Adam R, Donahue Manus J, Sills Allen K, et al. Alterations in default-mode network connectivity may be influenced by cerebrovascular changes within 1 week of sports related concussion in college varsity athletes: a pilot study. Brain Imaging Behav. 2016; 10(2):559-68. DOI: 10.1007/s11682-015-9407-3 [PubMed: 25972119]

55. Chen, Jen-Kai, Johnston Karen, M., Collie, Alex, et al. A validation of the post concussion symptom scale in the assessment of complex concussion using cognitive testing and functional MRI. J Neurol Neurosurg Psychiatry. 2007; 78(11):1231-8. DOI: 10.1136/jnnp.2006.110395 [PubMed: 17371902]

56. Lovell Mark R, Pardini Jamie E, Welling Joel, et al. Functional brain abnormalities are related to clinical recovery and time to return-to-play in athletes. Neurosurgery. 2007; 61(2):352-9. DOI: 10.1227/01.NEU.0000279985.94168.7F [PubMed: 17762748]

57. Sinopoli Katia J, Chen Jen-Kai, Wells Greg, et al. Imaging "Brain Strain" in Youth Athletes with Mild Traumatic Brain Injury during Dual-Task Performance. J Neurotrauma. 2014; 31(22):184359. DOI: 10.1089/neu.2014.3326 [PubMed: 24902051]

58. Dettwiler, Annegret, Murugavel, Murali, Putukian, Margot, et al. Persistent Differences in Patterns of Brain Activation after Sports-Related Concussion: A Longitudinal Functional Magnetic Resonance Imaging Study. J Neurotrauma. 2014; 31(2):180-8. DOI: 10.1089/neu.2013.2983 [PubMed: 23914845]

59. Gülin, Oz, Alger Jeffry, R., Barker Peter, B., et al. Clinical proton MR spectroscopy in central nervous system disorders. Radiology. 2014; 270(3):658-79. DOI: 10.1148/radiol.13130531 [PubMed: 24568703]

60. Lin AP, Liao HJ, Merugumala SK, et al. Metabolic imaging of mild traumatic brain injury. Brain Imaging Behav. 2012; 6(2):208-23. DOI: 10.1007/s11682-012-9181-4 [PubMed: 22684770]

61. Morgan Clinton D, Zuckerman Scott L, King Lauren E, et al. Post-concussion syndrome (PCS) in a youth population: defining the diagnostic value and cost-utility of brain imaging. Childs Nerv Syst. 2015; 31(12):2305-9. DOI: 10.1007/s00381-015-2916-y [PubMed: 26419243]

62. Parker Rolland S. Recommendations for the revision of DSM-IV diagnostic categories for comorbid posttraumatic stress disorder and traumatic brain injury. NeuroRehabilitation. 2002; 17(2): 131-43. [PubMed: 12082240]

63. Bouix, Sylvain, Pasternak, Ofer, Rathi, Yogesh, et al. Increased gray matter diffusion anisotropy in patients with persistent post-concussive symptoms following mild traumatic brain injury. PloS One. 2013; 8(6):e66205.doi: 10.1371/journal.pone.0066205 [PubMed: 23776631]

64. Bazarian Jeffrey J, Zhu Tong, Blyth Brian, et al. Subject-specific changes in brain white matter on diffusion tensor imaging after sports-related concussion. Magn Reson Imaging. 2012; 30(2):17180. DOI: 10.1016/j.mri.2011.10.001 [PubMed: 22079073] 


\section{Key Points}

- $\quad$ Routine clinical imaging is typically not indicated in sports-related concussion.

- $\quad$ Per the American Association of Neurology and the American Medical Society of Sports Medicine Guidelines, in concussion, imaging should only be performed if there are concerns regarding skull fracture, intracranial hemorrhage, or other intracranial pathology based on clinical exam.

- There are many studies today that may lead to important diagnostic and prognostic neuroimaging biomarkers that will assist in the diagnosis of brain alterations associated with concussion and that indicate those who are most at risk for adverse outcomes. This information will become available clinically over the next decade.

- Screening for clinical neuroimaging biomarkers will likely become important in ensuring adequate positive and negative predictive values and to maintain cost-effectiveness.

- While some neuroimaging exams may be appropriate in the acute phase of injury, many will likely be more appropriate in the subacute or chronic phases, and timing of scan will depend on clinical presentation. 


\section{Box 1}

\section{Imaging Protocols}

\section{Non-Contrast Clinical MRI Protocol for Concussion in Young Athletes}

- 3D T1-weighted magnetization prepared rapid gradient echo $(\mathrm{TR}=1520 \mathrm{~ms}$, $\mathrm{TE}=2.27 \mathrm{~ms}$, voxel size $=0.86 \mathrm{~mm}$ sagittal $\times 1 \mathrm{~mm}$ axial $\times 1 \mathrm{~mm}$ coronal, acquisition matrix $=256 \times 256$ )

- Axial T2 spin echo $(\mathrm{TR}=4300 \mathrm{~ms}, \mathrm{TE}=89 \mathrm{~ms}$, slice thickness $=2.5 \mathrm{~mm}$, acquisition matrix $=325 \times 512$ )

- $\quad$ Axial T2 FLAIR $(\mathrm{TR}=8000 \mathrm{~ms}, \mathrm{TE}=137 \mathrm{~ms}$, slice thickness $=4 \mathrm{~mm}$, acquisition matrix $=288 \times 320$ )

- Axial SWI $(\mathrm{TR}=27 \mathrm{~ms}, \mathrm{TE}=20 \mathrm{~ms}$, slice thickness $=1.25 \mathrm{~mm}$, acquisition matrix $=232 \times 256$ )

- $\quad$ DTI with tractography and TRACE (Directions $=35, \mathrm{~b}=1000, \mathrm{TR}=9500$ $\mathrm{ms}, \mathrm{TE}=88 \mathrm{~ms}$, slice thickness $=2 \mathrm{~mm}$, acquisition matrix $=128 \times 128)$

\section{Research Protocol in our Laboratory for Mild Traumatic Brain Injury}

- 3D T1-weighted magnetization prepared rapid gradient echo $(\mathrm{TR}=1800 \mathrm{~ms}$, $\mathrm{TE}=3.36 \mathrm{~ms}$, voxel size $=1 \times 1 \times 1 \mathrm{~mm}$, acquisition matrix $=256 \times 256$, flip angle $=7$ degrees)

- $3 \mathrm{D}$ T2-weighted fluid attenuation inversion recovery $(\mathrm{TR}=3200 \mathrm{~ms}, \mathrm{TE}=$ $456 \mathrm{~ms}$, voxel size $=1 \times 1 \times 1 \mathrm{~mm}$, acquisition matrix $=256 \times 256$ )

- DTI (Directions $=64, \mathrm{~b}=3000, \mathrm{TR}=13600 \mathrm{~ms}, \mathrm{TE}=111 \mathrm{~ms}$, slice thickness $=2 \mathrm{~mm}$, acquisition matrix $=256 \times 256$ )

- $\quad \mathrm{SWI}(\mathrm{TR}=30 \mathrm{~ms}, \mathrm{TE}=23 \mathrm{~ms}$, voxel size $=1 \times 1 \times 1 \mathrm{~mm}$, acquisition matrix $=256 \times 256)$ 


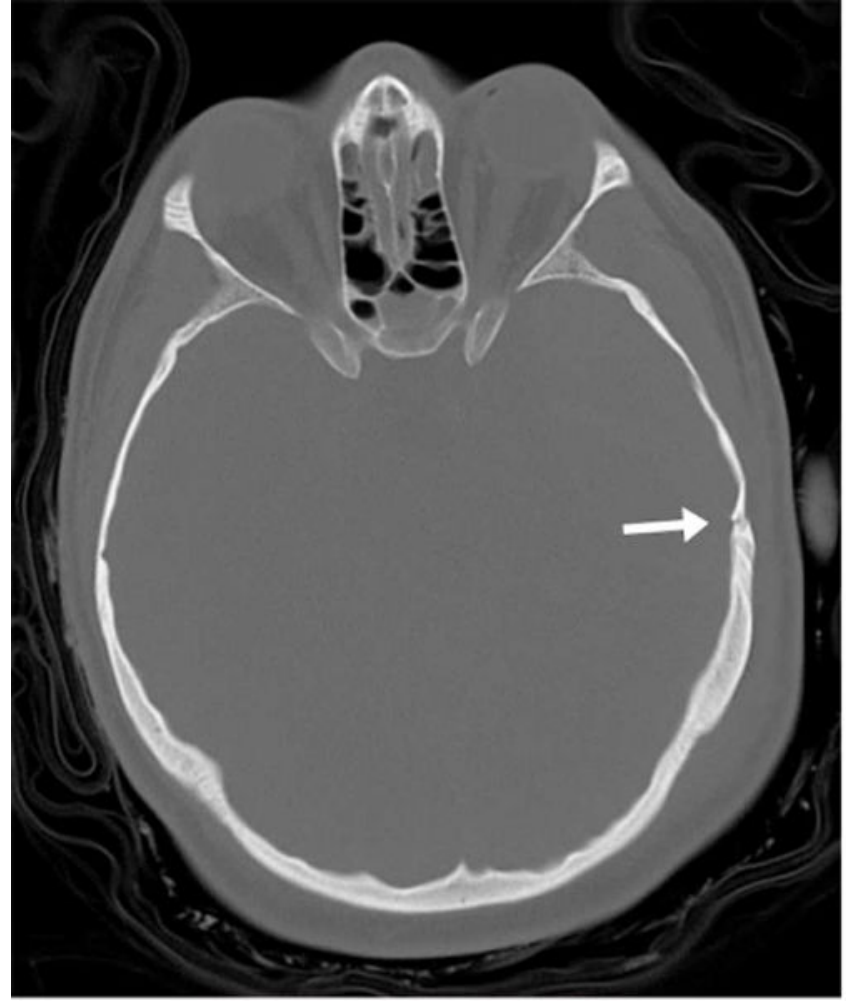

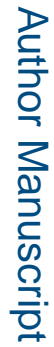

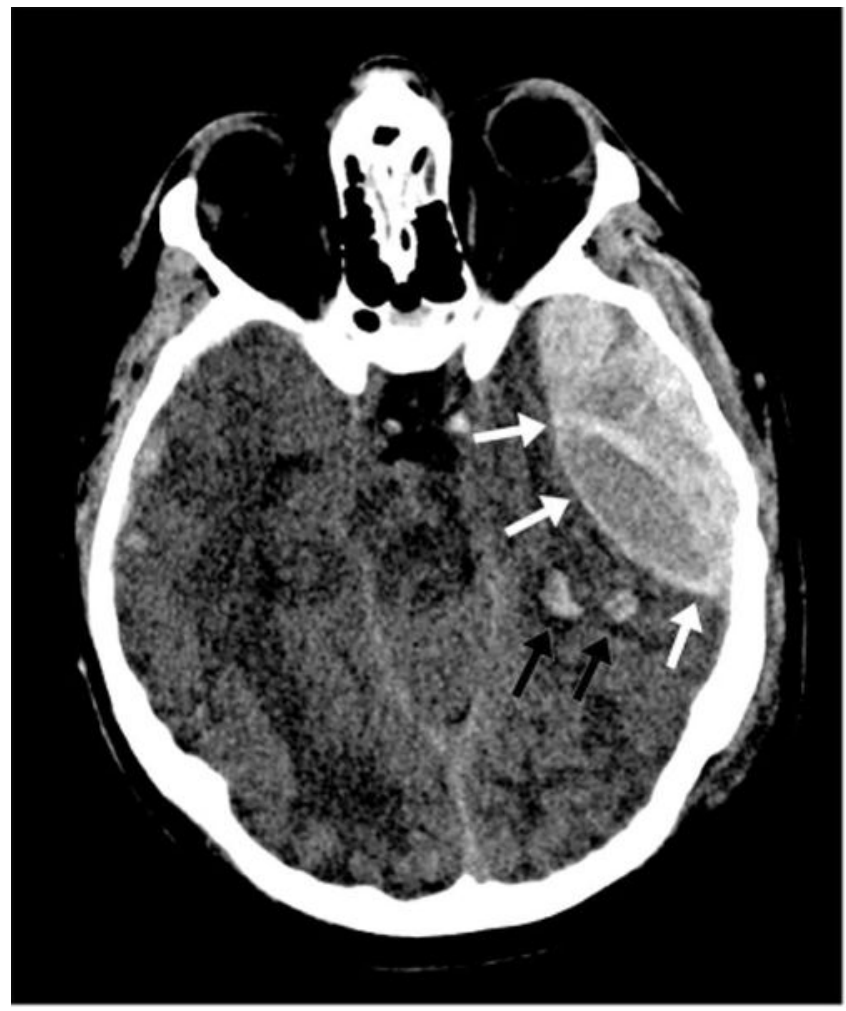

Figure 1. 
23 year-old male ejected from motor vehicle. (A) Axial CT image bone windows demonstrates a temporal bone fracture (arrow) in the region of the middle meningeal artery. (B) Axial CT image in soft tissue windows at the same level as Figure 1A demonstrates an associated large epidural hemorrhage (white arrows). Small foci of intraparenchymal hemorrhage are also visible (black arrows). (Images courtesy Liangge Hsu M.D., Brigham \& Women's Hospital) 


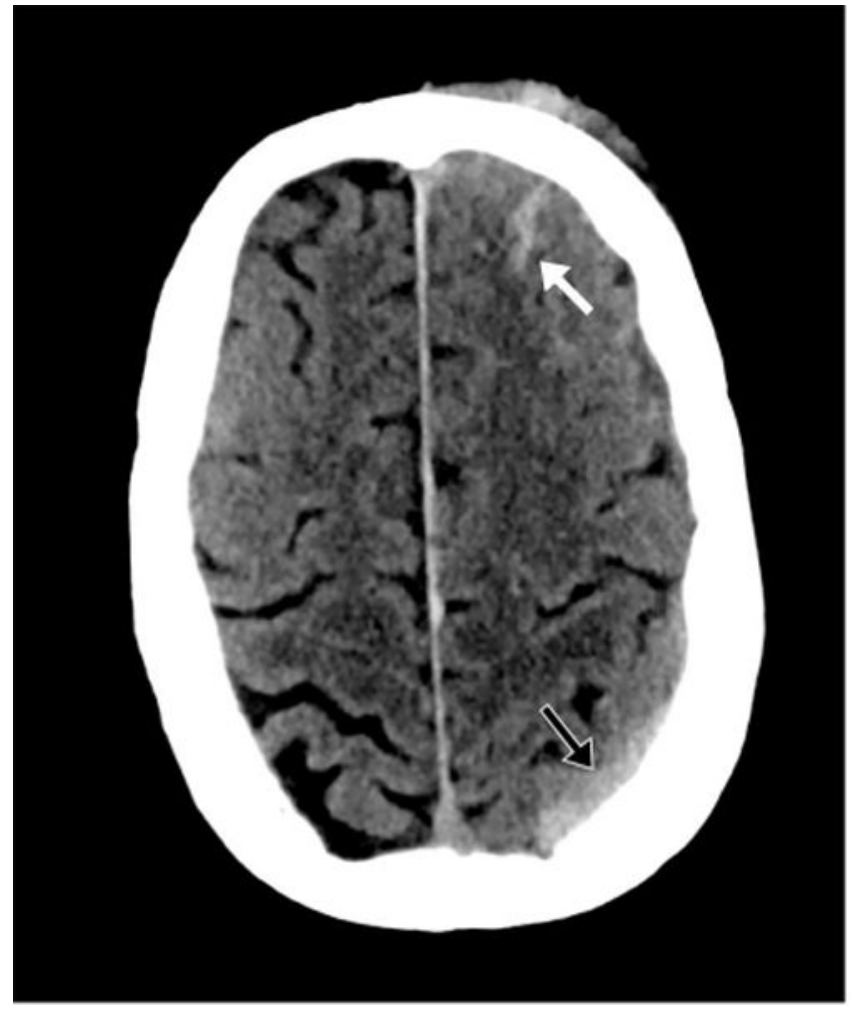

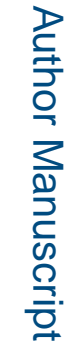

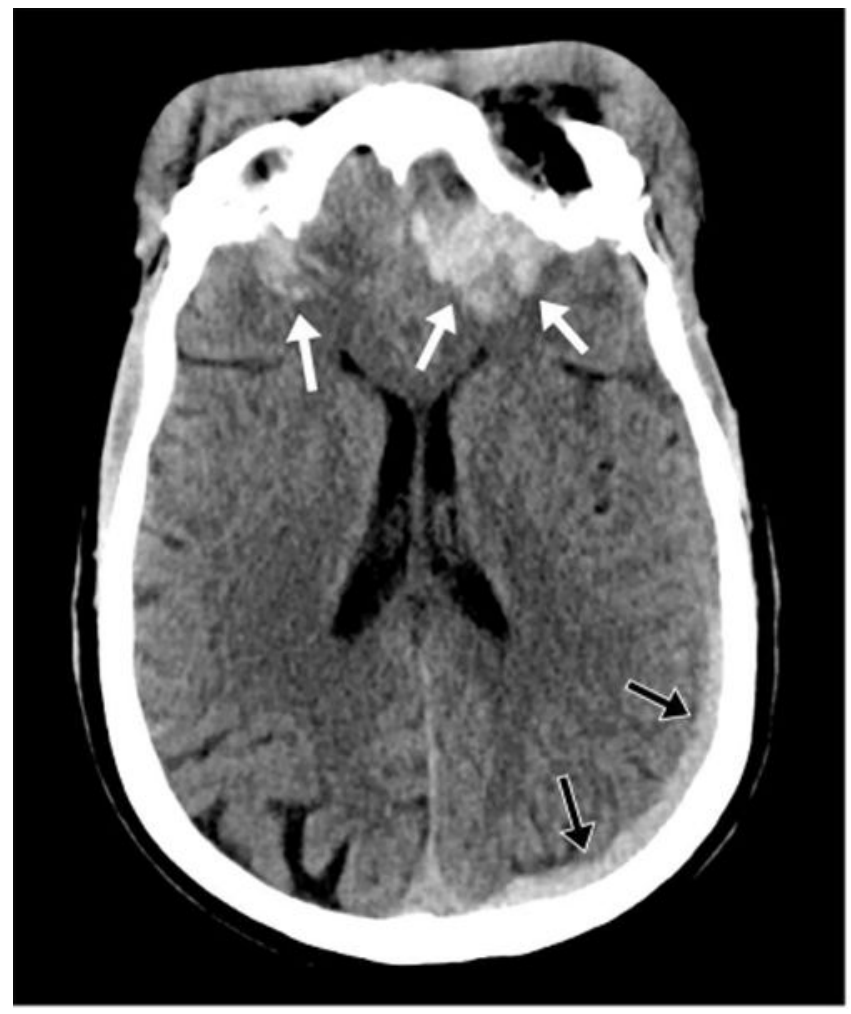

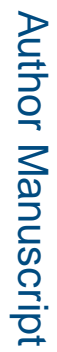




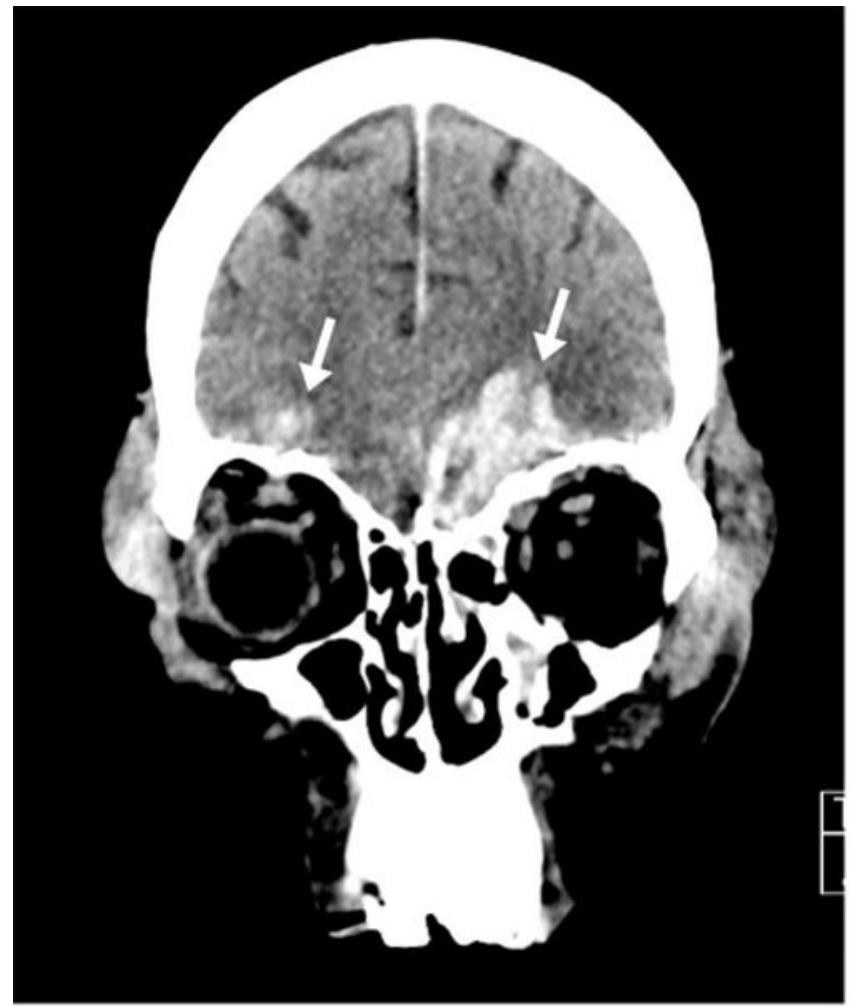

Figure 2.

46 year-old man status post 15 foot fall from roof. (A) Axial CT image in soft tissue windows demonstrates a left frontal subarachnoid hemorrhage (white arrow) and left subdural hemorrhage (black arrow). A left frontal subgaleal hematoma is also present. (B) Axial CT image in soft tissue windows, more inferior than Figure 2A, demonstrates bifrontal brain contusions (white arrows) and left subdural hemorrhage (black arrow). (C) Coronal CT image in soft tissue windows demonstrates the same bifrontal contusions (white arrows) clearly located along the floor of the anterior cranial fossa, a common location due to sudden traumatic compression of the brain in this region. 

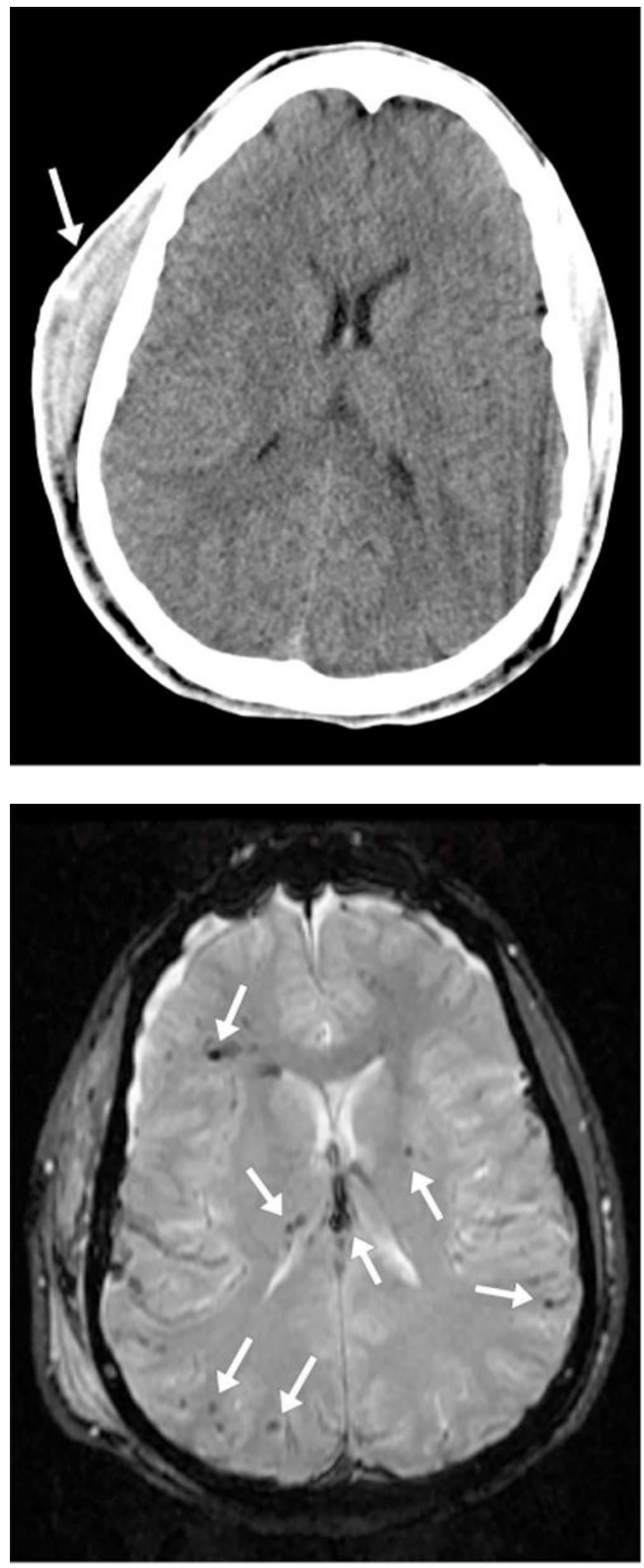

Figure 3. 
17 year-old male status post motor vehicle collision. (A) Axial CT image in soft tissue windows demonstrates a large right subgaleal hematoma (arrow) without any evident intracranial abnormality. (B) Axial gradient-recall echo (GRE) MR image in a similar plane demonstrates foci of susceptibility artifact (arrows), presumed to represent microhemorrhage, scattered along the gray white junction, in the right thalamus, in the left putamen, and in the fornix body. SWI has replaced GRE at our institution in most routine imaging protocols. (Images courtesy Liangge Hsu M.D., Brigham \& Women's Hospital) 

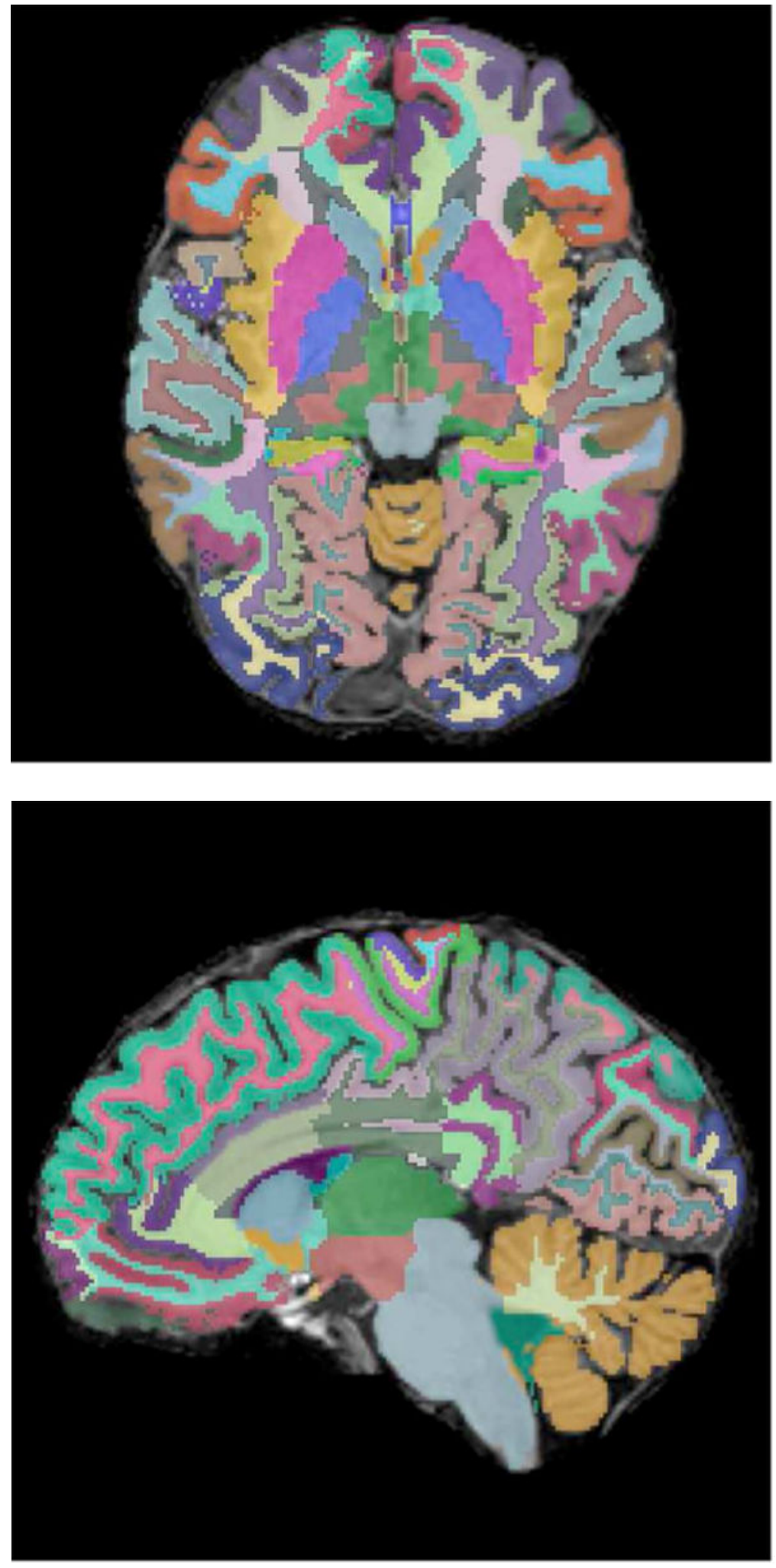

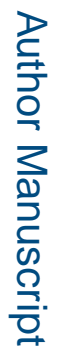




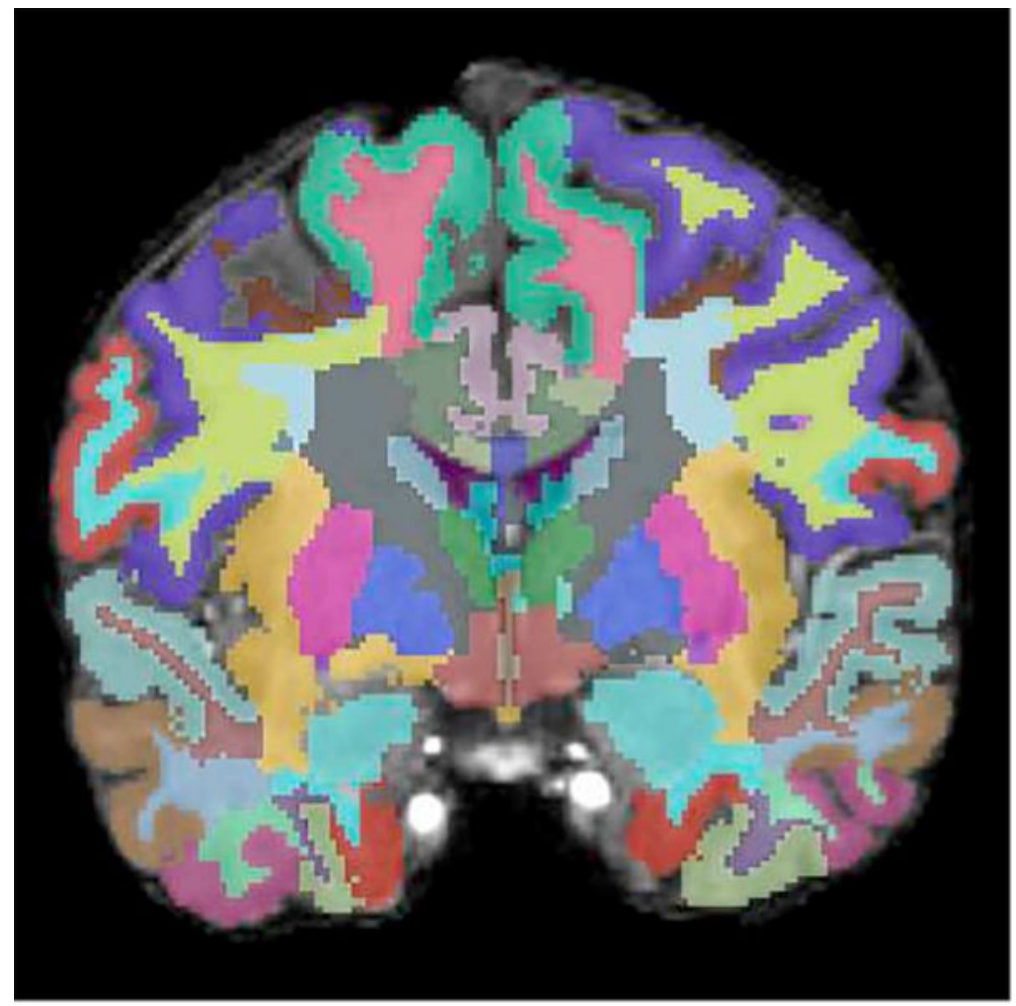

Figure 4.

(A) Axial, (B) sagittal, and (C) coronal images of the brain with automatic segmentation label maps generated by FreeSurfer superimposed on T1-weighted high resolution MR images. 

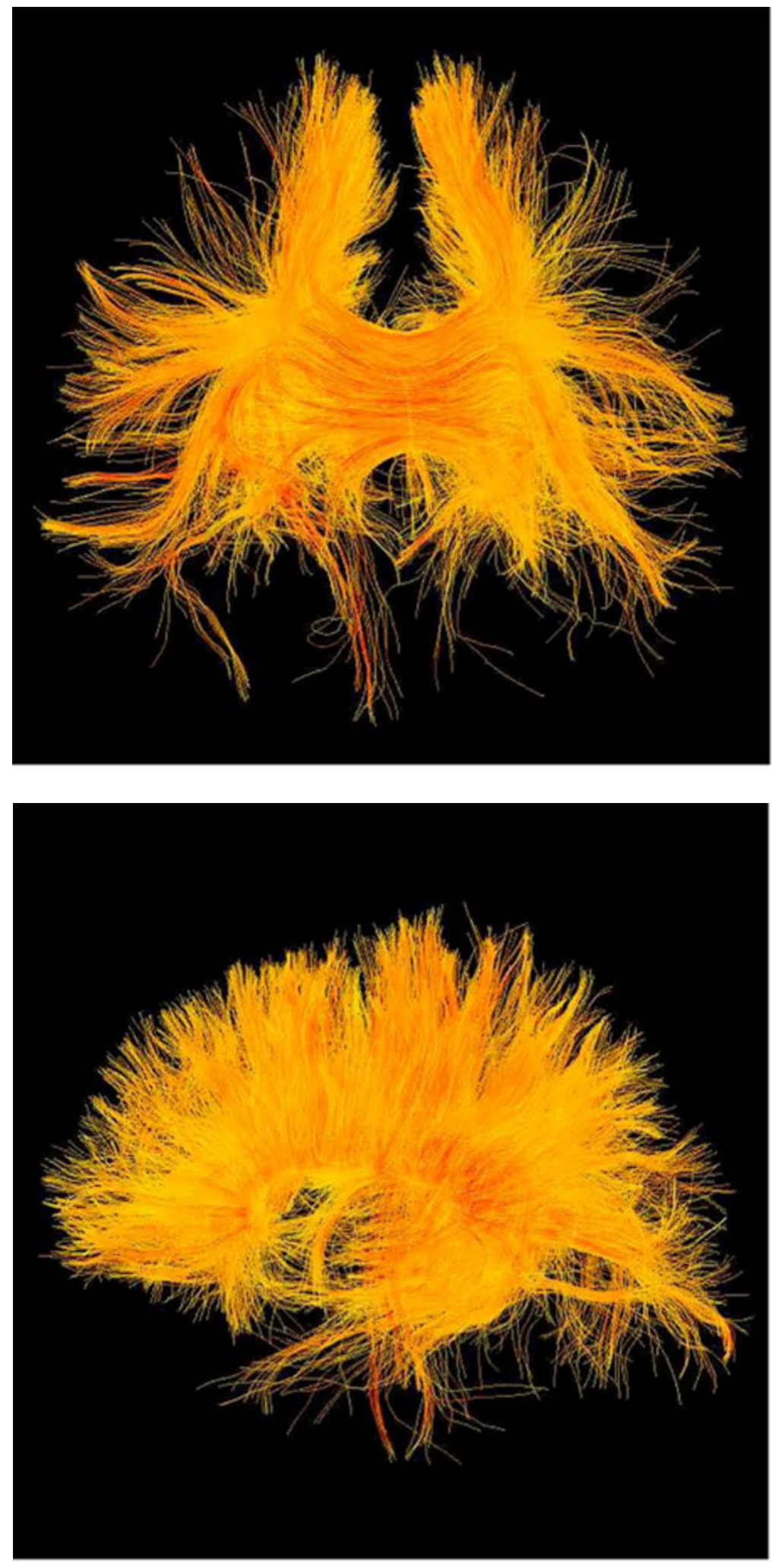

Neuroimaging Clin N Am. Author manuscript; available in PMC 2019 February 01. 


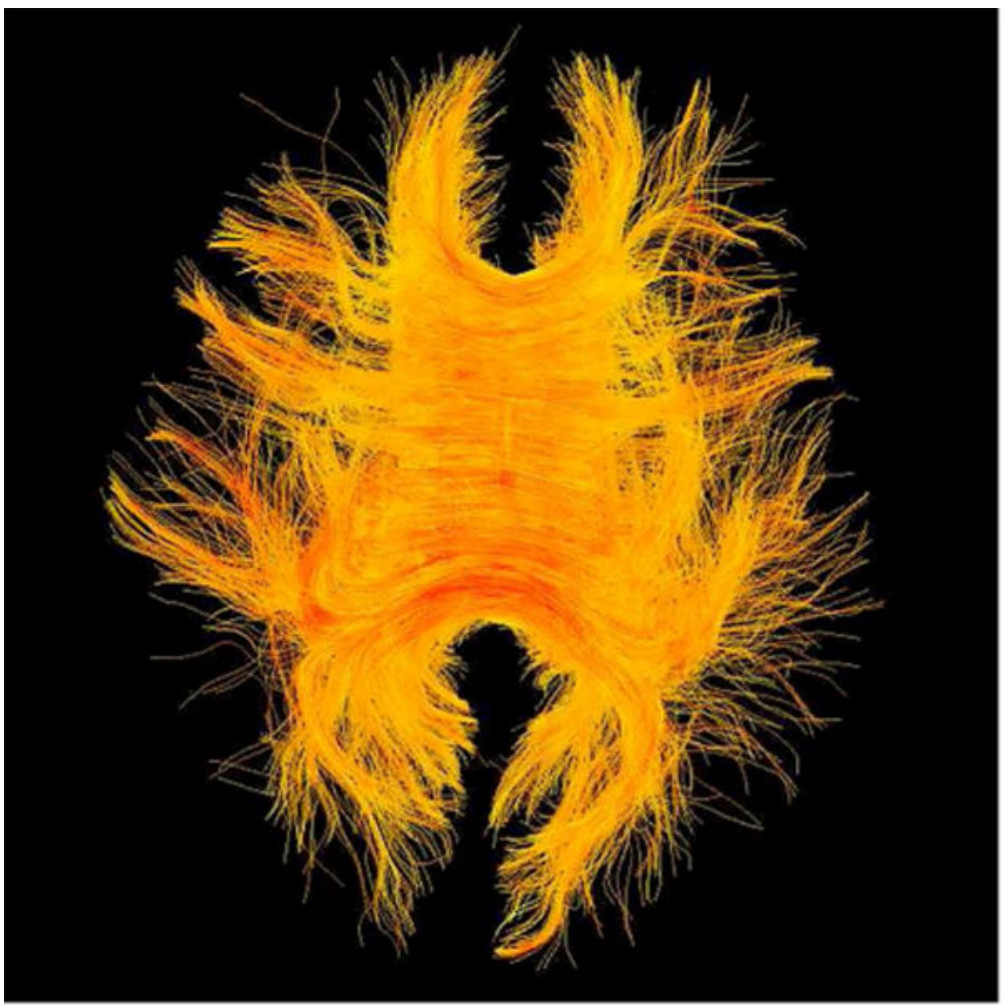

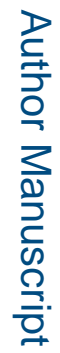

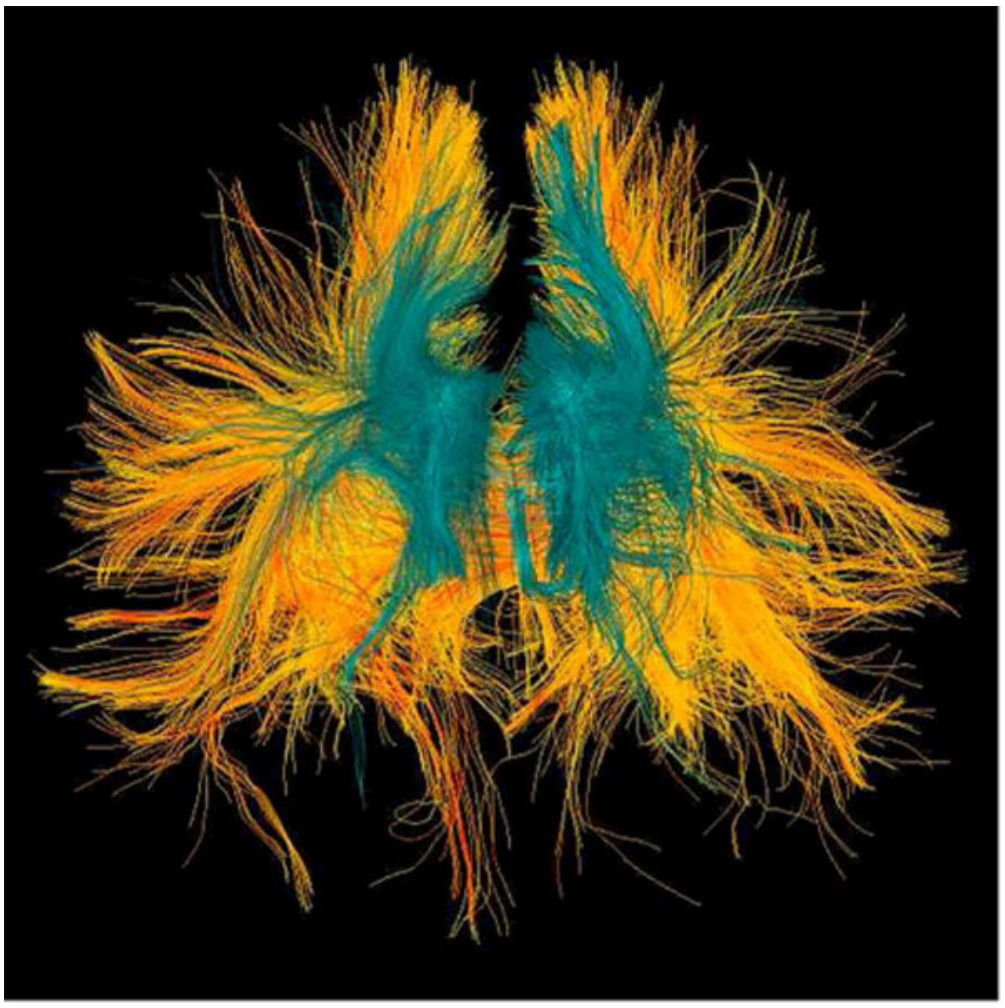

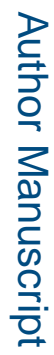




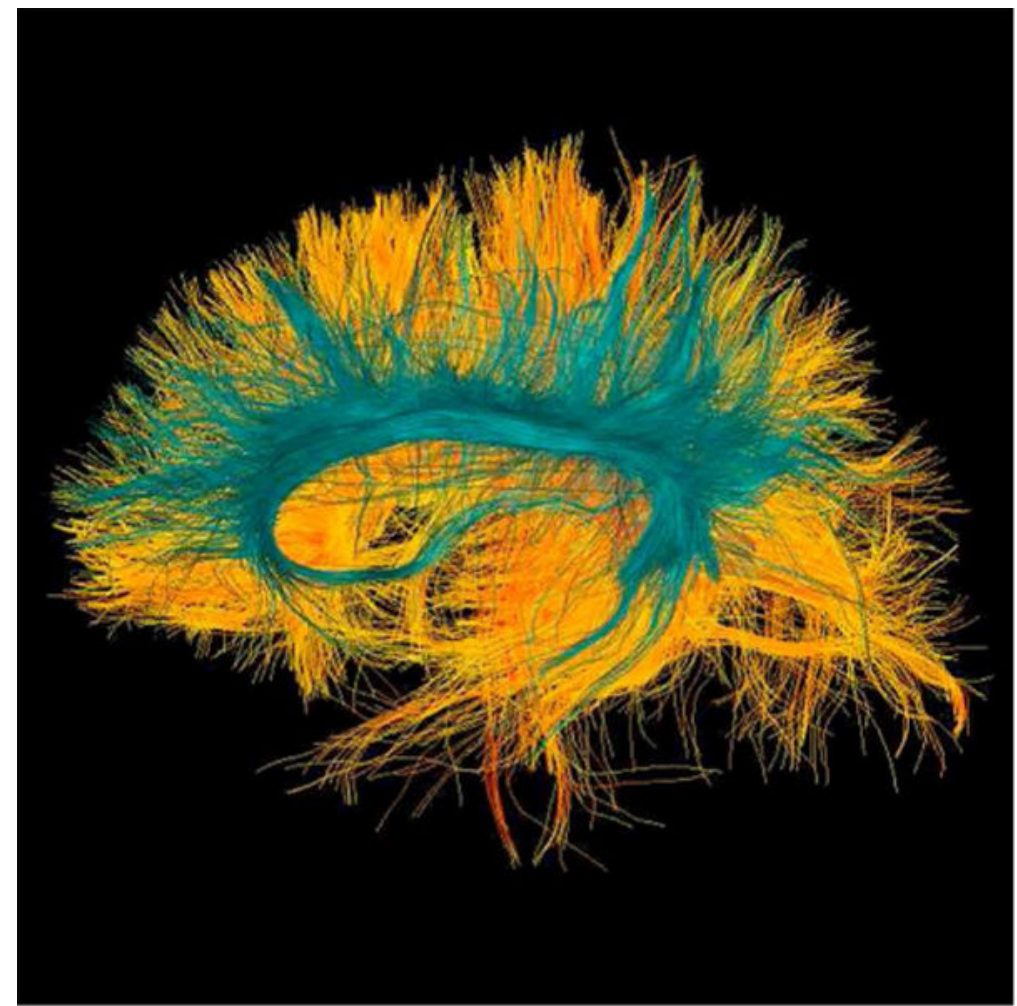

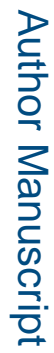

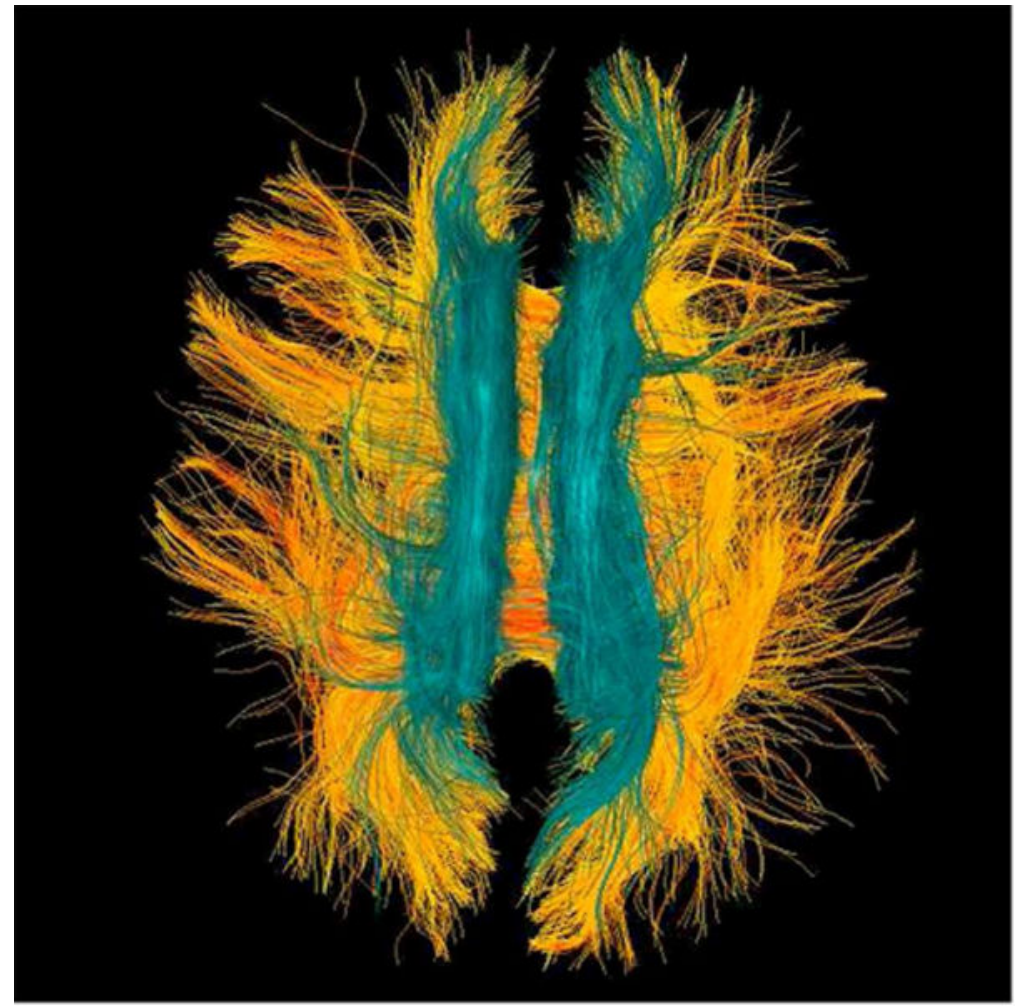

Figure 5. 
22 year-old male soccer player with history of concussion. (A) Frontal, (B) lateral, and (C) superior views of corpus callosum white matter fiber tracts generated with two-tensor tractography from diffusion-weighted images. The interhemispheric traversing fibers are particularly well demonstrated on the frontal and superior views. (D) Frontal, (E) lateral, and (F) superior views of corpus callosum (warm colors) and cingulate gyri (cyan spectrum) white matter fiber tracts generated with two-tensor tractography from diffusion-weighted images. Quantitative measures of the tracts are used to identify subtle white matter abnormalities not visible on conventional imaging. 